\title{
EthoHand: A Dexterous Robotic Hand with Ball-Joint Thumb Enables Complex In-hand Object Manipulation
}

\author{
Charalambos Konnaris ${ }^{1}$, Constantinos Gavriel ${ }^{2}$, Andreas A.C. Thomik ${ }^{1}$ and A. Aldo Faisal ${ }^{1,2,3}$, Member IEEE
}

\begin{abstract}
Our dexterous hand is a fundmanetal human feature that distinguishes us from other animals by enabling us to go beyond grasping to support sophisticated in-hand object manipulation. Our aim was the design of a dexterous anthropomorphic robotic hand that matches the human hand's 24 degrees of freedom, under-actuated by seven motors. With the ability to replicate human hand movements in a naturalistic manner including in-hand object manipulation. Therefore, we focused on the development of a novel thumb and palm articulation that would facilitate in-hand object manipulation while avoiding mechanical design complexity. Our key innovation is the use of a tendon-driven ball joint as a basis for an articulated thumb. The design innovation enables our underactuated hand to perform complex in-hand object manipulation such as passing a ball between the fingers or even writing text messages on a smartphone with the thumb's end-point while holding the phone in the palm of the same hand. We then proceed to compare the dexterity of our novel robotic hand design to other designs in prosthetics, robotics and humans using simulated and physical kinematic data to demonstrate the enhanced dexterity of our novel articulation exceeding previous designs by a factor of two. Our innovative approach achieves naturalistic movement of the human hand, without requiring translation in the hand joints, and enables teleoperation of complex tasks, such as single (robot) handed messaging on a smartphone without the need for haptic feedback. Our simple, under-actuated design outperforms current state-ofthe-art prostheses or robotic and prosthetic hands regarding abilities that encompass from grasps to activities of daily living which involve complex in-hand object manipulation.
\end{abstract}

\section{INTRODUCTION}

Our hands are considered to be one of the most complex end-effectors comprised by various types of joints, that require the synergistic movement of 27 bones and co-activation of 37 [1] muscles in order to function and explain observed hand kinematics [2]. The human hand's 24+ degrees of freedom (DoFs) allow for dexterous and precise tasks to be performed, with the least amount of effort. Human hand evolution drove our ability for complex manipulation of objects held within our hand, enabling object manipulation far beyond grasping mastered by other primates. We showed that the manipulative complexity of early flint stone tool making placed firm requirements on dexterity in early hominids [3]. Human evolution led to the distinct composite trapeziometacarpal saddle joint of the thumb setting us apart from non-human primates. This joint alone accounts for $40 \%$ [4] of a hand's dexterity, yet there was little development in

Brain \& Behaviour Lab - ${ }^{1}$ Dept. of Bioengineering \& ${ }^{2}$ Dept. of Computing, Imperial College London, South Kensington Campus, SW7 2AZ, London, UK, ${ }^{3}$ MRC Clinical Sciences Centre. Address for correspondence: aldo.faisal@imperial.ac.uk

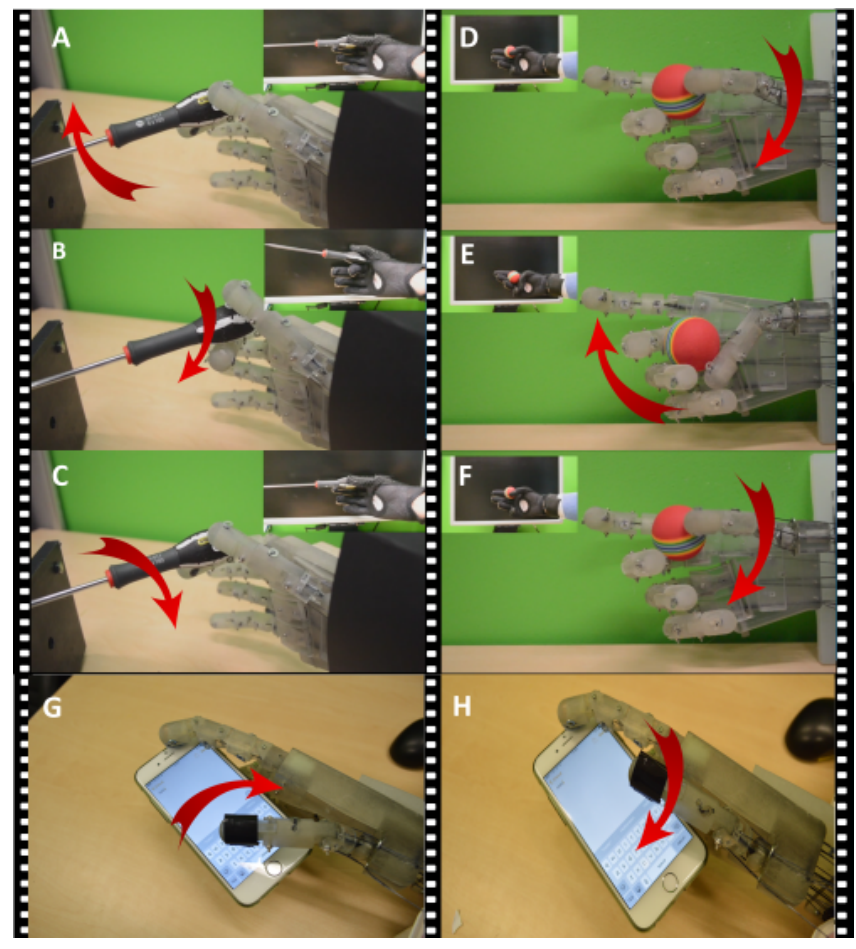

Fig. 1. Novel thumb and palm arch design enable the EthoHand to perform dexterous in-hand manipulation of complex objects. Subfigures A-C show the screwing motion in three steps. Subfigures D-F show the rotation of a softball making full use of our thumb and palm-arch articulations. Subfigures G\&H show the dexterity of our thumb by typing normal text on a smart phone (see also attached video)

robotics to improve the functional properties of the thumb joint.

The simultaneous action of multiple degrees of freedom of the fingers and thumb is fundamental, as we showed by analysing the principal components of human finger kinematics in evolutionary relevant behaviours (e.g. flint stone tool making[3]) or modern day activities (e.g.[2]). Emulating our hands' level of functionality in an anthropomorphic device has proven to be extremely difficult [5]. From a mechanical perspective, it is very challenging to integrate a large number of articulated DoF and corresponding actuators to manipulate the high-dimensional structure. Moreover, increasing the actuated DoF of robotic hands results in decreased overall grip strength and unstable systems. While the action of the 4 long fingers and simple grasp motions were successfully implemented in robotic designs, replicating the naturalistic thumb kinematics in artificial hands has not been realised.

Our thumb plays a crucial role while manipulating complex pre-held objects [6] as it covers a wide range of motion when engaged. Many robotic hands implement the thumb as 


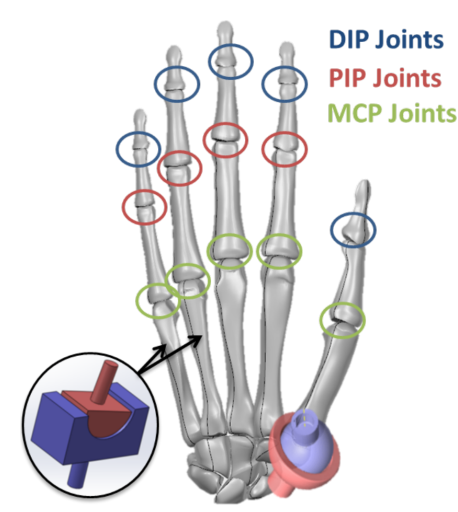

Fig. 2. Bones \& goints of the human hand. Our EthoHand introduces a ball in-socket articulation for the thumb to retain anthropomorphic kinematic capabilities for in-hand object manipulation. The palm-arch capabilities were mimicked by two artificial hinge joints on metacarpals $4 \& 5$ (circular inset and black arrows).

two hinge joints that are mounted off-axis with respect to the long fingers [7], to emulate the thumb capabilities, yet these causes un-naturalistic motions and the assumption that baseframe location movement is needed. Several commercially available robotic hands that utilise the double hinge joint feature for the thumb e.g.: Shadow Dexterous Hand, Gifu Hand, Anthropomorphic SAH Hand. Since there are no clear guidelines for the design of an anthropomorphic robotic hand and especially for the thumb [8], current state of the art robotic hands do not fully embrace the versatility of our thumb's motion.

Developing anthropomorphic end effectors is important, as it makes it easier for a human operator to map his natural manipulation behaviours and skills into commands for the device [9] - this is of fundamental importance in prosthetics and human robotics. Moreover, anthropomorphism improves safety and efficiency as human-like motion can be intuitively understood by humans [10] and may result in faster learning and lower abandonment rates in the case of active prosthetics. The complex biomechanical and neural architecture of the hand poses challenging questions for understanding the control strategies that underlie the coordination of finger movements, ranging from multi-digit grasping to the individuated movements of single digits [11]. It is therefore crucial to understand together the interaction between biomechanical architecture and neurobiological function of the hand before attempting to replicate it in a robotic hand [2].

\section{DESIGN \& MECHATRONICS}

In order to perform a simple action such as manipulating a ball, several complex neural, mechanical and sensory interactions take place. Before going into the design phase, we must first address the topic of what characteristics make the human hand so dexterous and versatile; capable to adapt and perform complex action manifolds. The human hand has the ability to dynamically move its fingers base frame locations allowing it to perform a wide range of prehensile grasps and in-hand manipulation. Commercially available prosthetic hands are able to attain a large majority of activities of daily living (ADLs) only via static postures, while there is little systematic comparative data on robot hand capabilities. We aimed to design a dexterous robotic hand that is capable of performing the 33 most commonly used grasps (see [12]) of activities of daily living and more importantly in-hand object manipulation.

Replicating the grasp functionality of digits 2-5 (index, middle, ring and pinkie respectively) has been achieved in the early stage of artificial hands, because they consist of relatively simple hinge joints: distal interphalangeal, proximal interphalangeal and metacarpophalangeal (DIP, PIP, MCP respectively, see figure 2 ). In order to replicate the dexterity and agility of the real hand in an artificial environment, we choose to design a robotic hand that consists of 24 DoF. The unique feature of the human hand that allows for precise and complex in-hand manipulation is our thumb. To this end, we focus on the development of a novel thumb articulation. The size of our artificial hand was calculated based on the average dimensions of a male person using anthropomorphic tables [13]). Using rapid prototyping, the artificial hand was 3D printed using standard ABS plastic material (see photo in Figures 1 and 4) weighing a total of 190 grams.

a) Long fingers: The first step in the design phase was the development of digits 2-5. All fingers were designed as hollow cylinders consisting of 3 joints: DIP, PIP and MCP (see Figure 3, sub-figure A). Pulleys were implemented at each joint location to prevent tendons moving away from the central axis of the finger, minimising friction during motion. Attachments for tendons were created midway through each phalanx in order to utilise maximum leverage when actuating each phalanx. Tendons on each phalanx were secured using external ring supports (see sub-figure A in figure 4). Digits 2-5 were designed to reproduce the same kinematic motion ranges as in the real hand: flexion/extension and adduction/abduction (see Table I for ranges of motion for each joint).

b) Palm and Movable-arch: We introduced two additional joints in the dorsal region of the palm (see Figure 2.C \& 3.A.C), aiming to improve in-hand manipulation and provide secure grasps in various static postures. These additional DoFs enable digits 4 and 5 to fully flex providing agility when performing dynamic action manifolds such as rotating a ball where palm arch opposition as shown in Figure 4.A,D (see also supplementary video). Since the goal of our overall hand project is to develop a fully functional artificial hand, supplementary wrist attachments were created for future purposes on the proximal part of the palm (see Figure 3.C).

c) Ball-jointed Thumb: In order to develop a dexterous anthropomorphic artificial hand with conformal thumb ca-

TABLE I

ETHOHAND RANGES OF JOINT MOTION

\begin{tabular}{|c|c|c|c|c|c|}
\hline Joint & MCP & PIP & DIP & OPPOSITION & CMC \\
\hline Digit 4\&5 & $\pm 25^{\circ}$ & $0-90^{\circ}$ & $0-90^{\circ}$ & $-15+35^{\circ}$ & - \\
Digit 2\&3 & $\pm 25^{\circ}$ & $0-90^{\circ}$ & $0-90^{\circ}$ & - & - \\
Thumb & $0-90^{\circ}$ & - & $0-90^{\circ}$ & - & \pm 65 \\
\hline
\end{tabular}



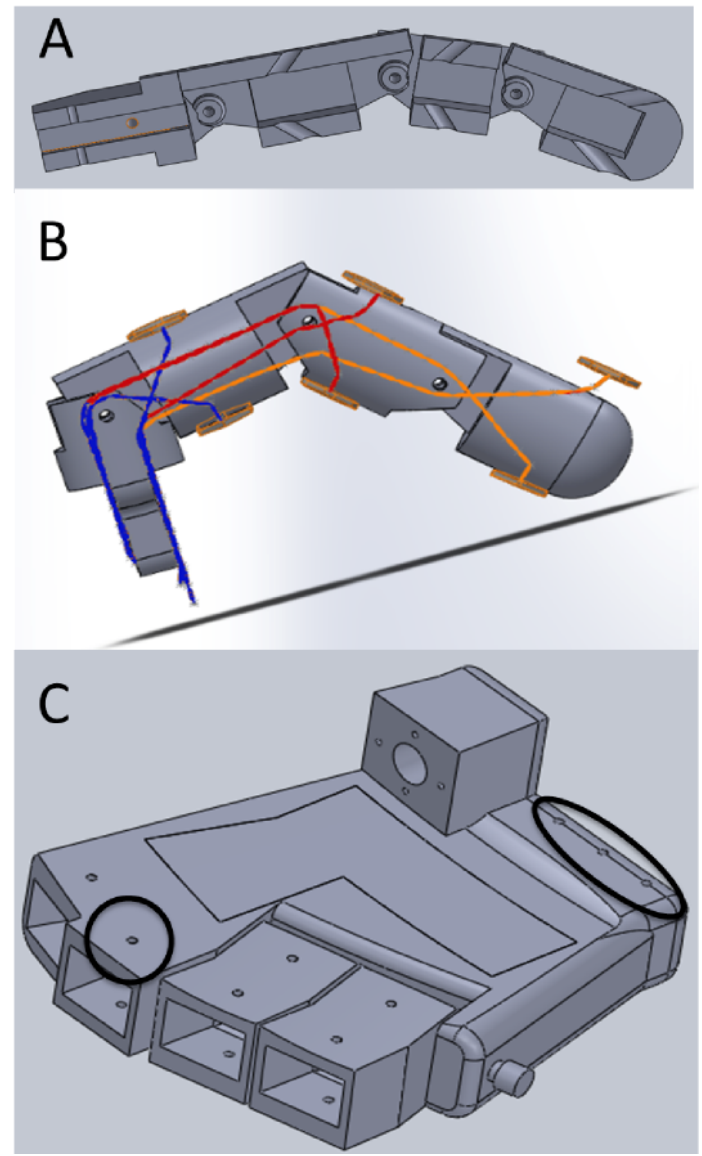

Fig. 3. Elements of EthoHand 3D printed design: (A) Cross-section of index finger. The holes were created midway each phalanx in order to exert the most leverage in both flexion and extension. (B) Wiring of tendons in the index finger (tendons are blue, green, red lines with attachment points). This wiring configuration requires the least amount of torque by the motors. (C) Palm design without attached fingers. The individual palm-arch joints for digits 4 and 5 are in the centre. The ellipse indicates 3 holes to allow for forearm attachment.

pabilities, a ball joint was introduced to improve the thumb articulation. Our thumb articulation consists of two parts: 1) the ball joint where DIP and MCP phalanges are attached and 2) the socket, which is secured on the palm (see Figure 4.C). A "ball-in-socket" joint comprises 3 DoF: two rotational DoF, specifying a directional axis that allows for planar movements and an additional DoF, rotation about the directional axis. A smart physical control of this particular joint was developed in order to avoid rotation of the finger along its central axis by using two pairs of antagonistic tendons as shown in figure 4.E. Moving the thumb to a particular end-point location requires the synergistic movement of the two tendon pairs.

\section{EthoHand Actuation \& CONTROL}

The robotic hand was under-actuated using 7 motors to control 20 out of $24 \mathrm{DoF}$ in an intuitive manner by coupling DIP, PIP and MCP joints. In order to test the kinematic and functional capabilities of the device, the motors were mounted on an external support acting as the forearm. Tendons were attached midway on each phalanx and the other end was directly mounted on standard sized servo motors (HighTech HS-422, Hitech RCD Inc., Poway, CA) using springs to avoid tendon slack. For digits 2-5, DIP, PIP and MCP joints were coupled together, thus each long finger was actuated via one motor, which allowed for flexion and extension. The two additional artificial joints that allowed for palm-arch capabilities were coupled on the corresponding MCP joints of digits $4 \& 5$. At this stage, the DoF responsible for adduction/abduction for digits 2-5 were physically blocked. The thumb was fully actuated using 3 servo-motors: 1 motor for the red set of tendons, 1 motor for the orange set of tendons (see figure 4.A.E, \& figure 6.B) and 1 motor to control flexion/extension. All servo-motors were connected to a micro-controller (Arduino Mega 2560, Arduino, Italy) with an external battery supply (4 x AAA) to satisfy the voltage and current requirements of both the EthoHand servos and the micro-controller.

Controlling the high-dimensional structure of the artificial hand in an intuitive effortless manner is still an unsolved issue in the context of robotic tele-operation and prosthetics. As a first step, a graphical user interface (GUI) was developed in Matlab, allowing for manual joint-by-joint control. The GUI included a range of pre-defined grasps (see figure 5 and video). Using the GUI to operate multiple DoF proved to be time-consuming and unintuitive. Additionally, when using a pre-defined posture to grasp an object, manual adjustments of joints were required to avoid slippage of the held object.

We choose to actuate our hand by streaming, real-time joint angles from 22 sensors embedded in a dataglove (CyberGlove III, CyberGlove Systems LLC, CA, USA). Using a CyberGlove to control the developed hand, allowed the user to exploit visual feedback to learn kinematic motions of appropriate digits. This in turn, compensates for the inability of the artificial hand to move base-frames locations when manipulating pre-held objects. Digits 2-5 were calibrated to zero when fully extended while the thumb's zero location was set at the joint's central axis. Using one to one mapping for digits 2-5, each joint of the artificial hand was receiving real-time data from the corresponding sensor on the CyberGlove allowing for flexion and extension. For the thumb, where the CMC joint is actuated by 2 servos, the two sensors on the CyberGlove that measure adduction/abduction were mapped on the 2 motors.

\section{Dexterous Manipulation of Objects}

a) Grasping Objects: : The first step taken to visually represent the dexterous capabilities of the robotic hand was to perform the 33 most commonly used grasps in activities of daily living from the GRASP project [12]. The grasp taxonomy classifies grasps according to: opposition, virtual finger assignments, types in terms of power and lastly according to the position of the thumb. In figure 5, we show the sub-category of the 8 most critical grasps in activities of daily living, also performed by all state of the art myoelectric prostheses (see also attached video). Given the mechanical robustness and reliability of the EthoHand to hold objects of daily life using all 5 digits we limited it to objects that weigh less than $2.3 \mathrm{~kg}$. 


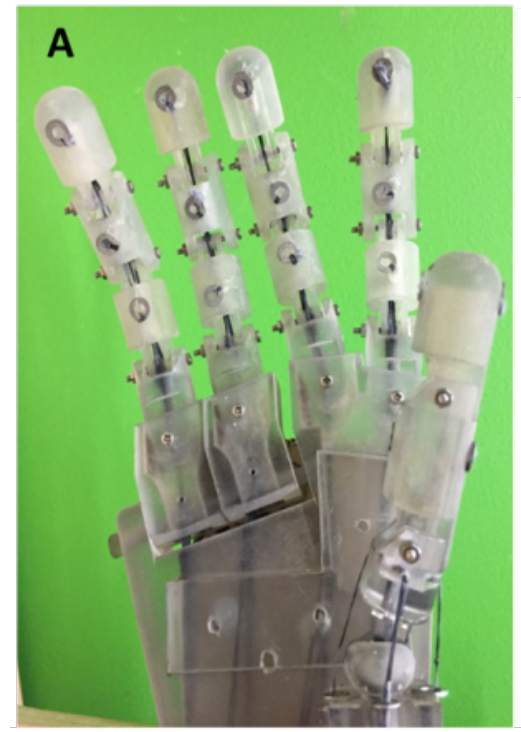

Ball Joint Control:

Pair of Agonist and Antagonist Tendons

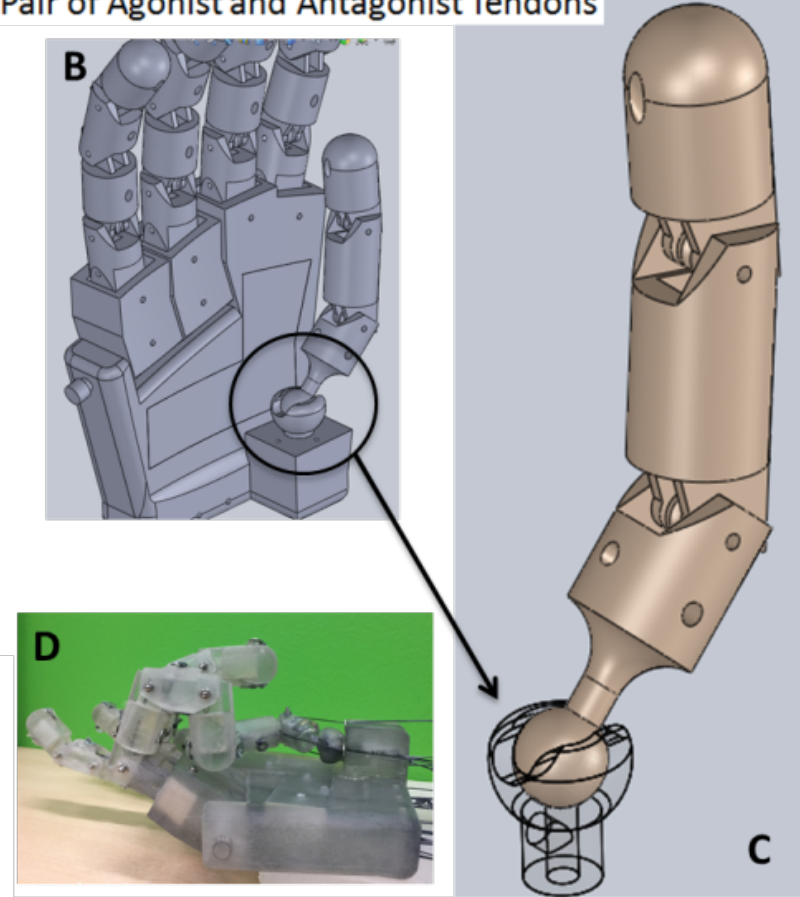

Tendons responsible for planar Flexion/ Extension

Tendons responsible for planar Adduction/Abduction

Fig. 4. (A) Photography of the EthoHand. (B) Placement of thumb articulation in palm structure. (C) Thumb ball joint in close up, the socket of the ball joint is rendered transparent. (D) Palm-arch capability through adding an extra joint to fingers 4 and 5. (E) Physical actuation of the thumb through antagonistic tendons inside the ball joint for spherical control: (red) planar Adduction/Abduction (orange) planar Flexion/Extension.

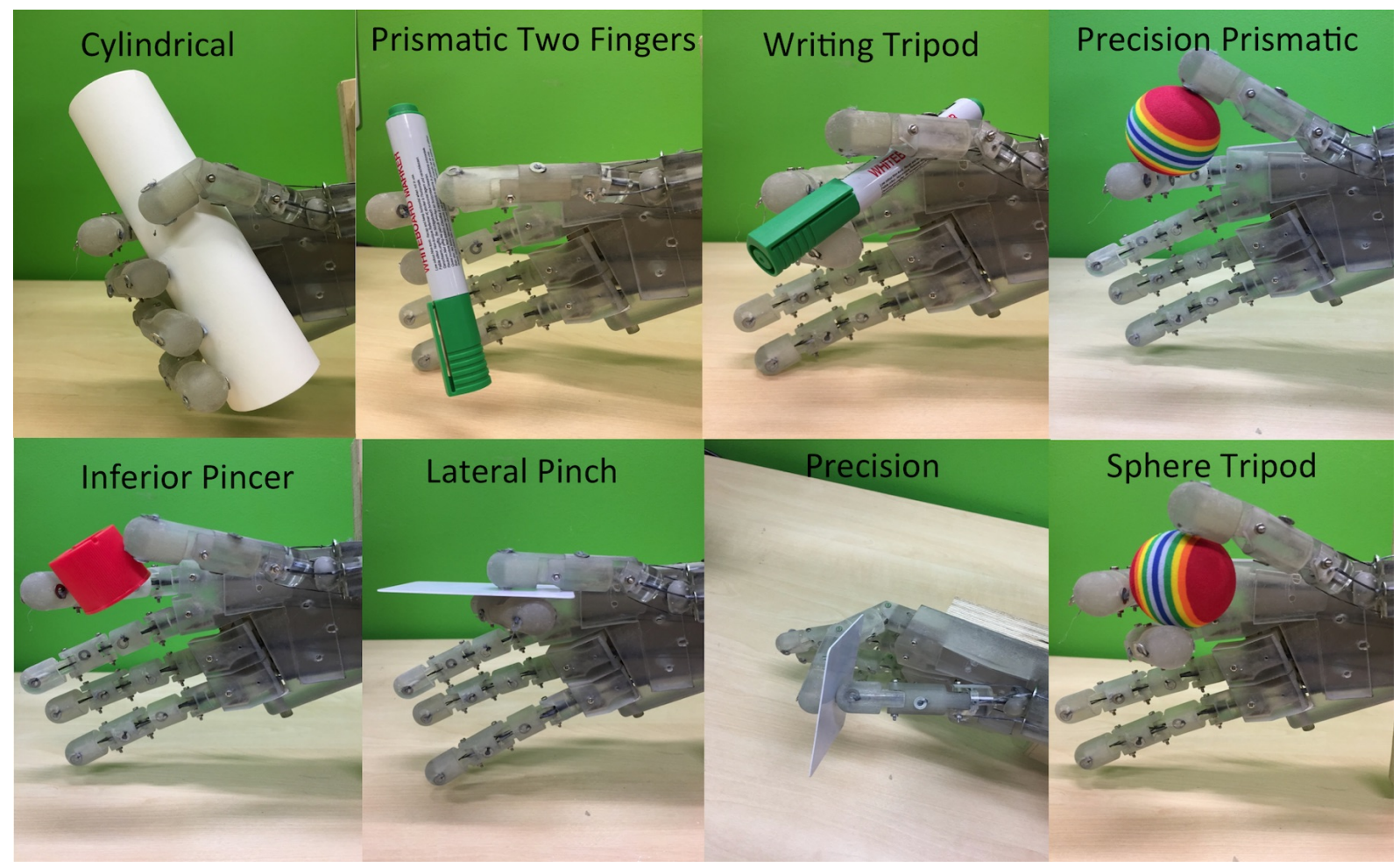

Fig. 5. Demonstration of dexterous grasping capabilities of our hand with in-hand object manipulation exercised through teleoperation with a Cyberglove (see also attached video). The 8 most commonly used grasps in activities of daily living [12]. 
b) In-hand Manipulation of Objects: One of the primary aims of this project is to introduce the naturalistic concept of in-hand manipulation. In this direction, the robotic hand was challenged to replicate and perform novel moves that current state of the art prosthetic and robotic hands lack. Manipulating pre-held objects in real life requires precise simultaneous control of multiple digits and sensory feedback interaction. In the case of prosthetic hand users, visual feedback is primarily used. Using CyberGlove the EthoHand was able to mimic 4 novel in-hand manipulations such as palm-arch opposition, rotate a pre-held ball, rotate a screwdriver and precise tapping on the screen of a mobile device (see Figure 1 and video). By performing these moves we show the precision and fine dexterous capabilities of our thumb's design.

c) Dexterity Evaluation \& Comparison: We evaluate the dexterity of our hand, in the context of human anthropomorphism, i.e. the ability of an artificial device to mimic and execute action manifolds in a human-like manner. We measure the dexterity level of our hand following [14], which uses the geometry of convex hull of the workspace of each digit as a measure. The metrics of this approach are based on the relative coverages of human and robot finger phalanges workspaces as well as human and robot finger base locations workspaces. This particular procedure of assessing the anthropomorphism of robot hands, takes into account not only the end-point of each finger but also the configuration. We have compared our novel articulation of the thumb and digit 2 of our artificial hand with a state-of-the-art robotic hand (Shadow Hand, Shadow Robots Ltd, London, UK) (see Table II for results). We take the dexterity measure further by collecting physical manipulation data using optical motion capture systems (Flex 13, Optitrak, Natural Point Inc., Corvallis, OR), by attaching 3 markers on the three phalanges of the thumb and digit 2 (see Figure 6).

Table II shows that our novel articulation of the thumb results in a level of anthropomorphism of $57 \%$, whereas the Shadow Hand scores 19\%. We note that evaluation of dexterity with physical kinematic data shows a 5\% drop in workspace volume for the thumb when compared with the simulations. This drop of percentage denotes that mechanical noise such as friction or slack in the tendons is present, preventing the ball-joint articulation to move to its predesigned range of motion. We propose strongly that in general robotic hand evaluations should use independent position measurements over simulated motions or internal sensors to validate performance capabilities.

\section{DISCUSSION}

Our novel design of the thumb joint enabled our developed hand to execute the 33 most commonly used grasps in activities of daily living. Moreover, we have demonstrated control of precise and complex in-hand manipulation tasks such as texting on a hand-held mobile phone, without requiring movable joints locations nor sensory tactile feedback in tele-operation. To demonstrate improvement a systematic comparison across disciplines is required to act as a scientific robust method for comparing functional abilities of artificial hands and human hands. This sets the baseline foundation for evaluating engineering improvement. We therefore quantified the dexterity and anthropomorphism of our hand design with respect to human, prosthetic and robotic hands setting a cross-disciplinary precedent that spans the field of biorobotics. We demonstrated that our design doubles dexterity measures beyond those of existing hand designs (at least for those cases where such data was actually published).

The ball-jointed thumb proves to be superior in range of motions and daily-life activities over current prosthetic and dexterous robotic hands [15]. These use a common design for thumb articulation: the double hinge joint, where one hinge joint stacked and 90 degrees rotated on another hinge joint. This limits the artificial thumb to perform only single plane flexion/extension. The double hinge joint design is deployed in prosthetics where if the user wishes to switch from a cylindrical to lateral grasp the end-user has to physically readjust the thumb joint to the appropriate plane. This leads to the end-user assisting his own device instead of the other way round. By introducing our novel ball-joint thumb articulation in the form of a ball joint, we enabled the robotic prosthetic hand to perform naturalistic, multi-planar movements similar to the human thumb.

We operated the EthoHand in direct tele-operation mode and enabled complex in-hand manipulations without any tactile or haptic feedback, simply by mapping human hand motions from Cyberglove to finger joints (the user had only visual feedback). Utilising the novel design of our thumb, we successfully executed novel dynamic moves that require simultaneous control of multiple digits, such as rotating a ball, rotating a screwdriver and precise tapping on the screen of a mobile device. Our improved anthropomorphic hand enables us to deploy in a straightforward manner to deploy neural engineering and tele-operation control schemes derived from natural hand movements to actuate $24 \mathrm{DoF}$ hands [16]. To achieve advanced in-hand manipulation tasks, robotic hands are required to be equipped with distributed tactile sensing. This enables them to continuously provide information about the magnitude and direction of forces at all contact points between them and the objects they are interacting with [17].

It is commonly assumed that in-hand object manipulation

TABLE II

THUMB DEXTERITY EVALUATION BASED ON [14], WHERE 100\% SUGGESTS FULL CAPABILITIES OF HUMAN HANDS.

\begin{tabular}{|c|c|c|c|}
\cline { 2 - 4 } \multicolumn{1}{c|}{} & \multicolumn{2}{c|}{ Simulation } & $\begin{array}{c}\text { Kinematic } \\
\text { Tracking }\end{array}$ \\
\cline { 2 - 4 } \multicolumn{1}{c|}{} & Shadow Thumb & Etho Thumb & Etho Thumb \\
\hline Proximal Phalanx & $15 \%$ & $56 \%$ & $49 \%$ \\
Middle Phalanx & - & - & - \\
Distal Phalanx & $22 \%$ & $56 \%$ & $50 \%$ \\
\hline Total & $19 \%$ & $56 \%$ & $50 \%$ \\
\hline & Shadow Finger & Etho Finger & - \\
\hline Positions & $34 \%$ & $41 \%$ & - \\
Orientations & $60 \%$ & $62 \%$ & - \\
\hline Total & $47 \%$ & $51 \%$ & - \\
\hline
\end{tabular}




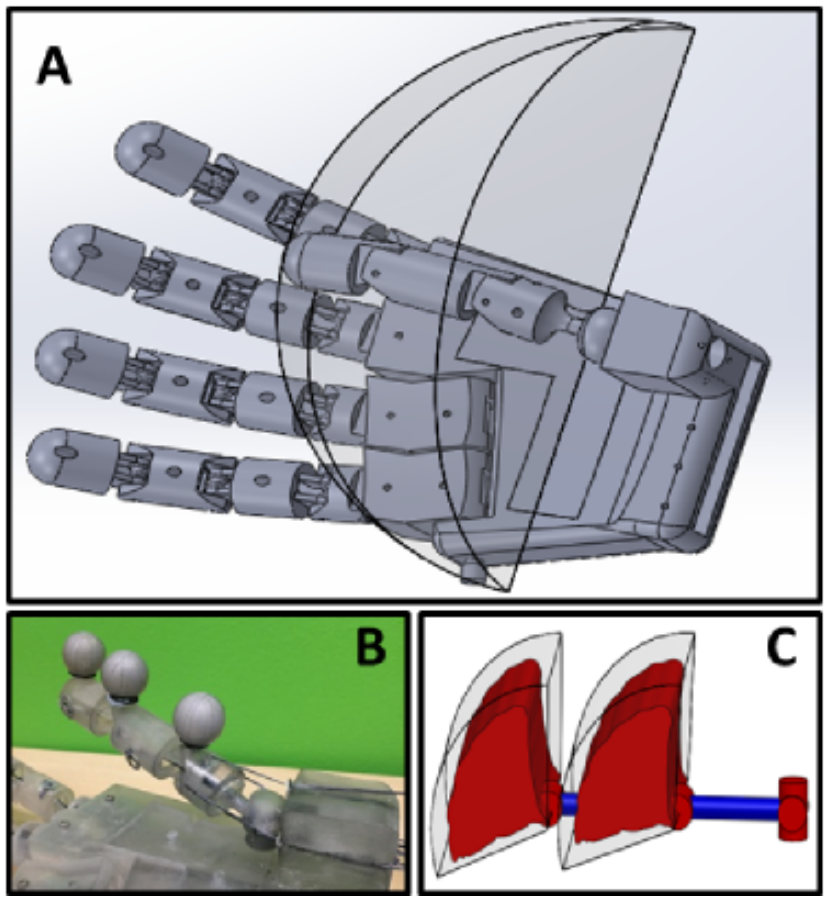

Fig. 6. Active workspace of the EthoHand thumbs. Sub-figure (A) shows the active workspace of the thumb in CAD. Sub-figure (B) shows the placement of optical markers (silver spheres) on the EthoHand phalanges. Sub-figure (C) shows the workspace computation we obtained by computing the convex hulls from the data. Grey shades denote the convex hull of simulation data. Red shades depict the convex hull from tracking data.

requires the use of a. moveable joint frames and b. proprioceptive and tactile feedback: Specifically, the unique conformal capabilities of the human hand to move base-frame locations, i.e. the locations of the joints with respect to each other [14], even perhaps a fully flexible palm [18]. Moreover, the lack of proprioceptive feedback is commonly attributed to the limited dexterity experienced by prosthetic and teleoperation users [19]. However, here we demonstrated that complex in-hand manipulations are already enabled by our ball-jointed thumb - even by using an under-actuated design. This simple and under-actuated design outperforms current state-of-the-art prosthetic or robotic hands in terms of abilities that encompass from grasps to activities of daily living [20], which involve complex in-hand object manipulation such as rotating and translating objects in-hand or texting on a smart phone.

\section{REFERENCES}

[1] H. van Duinen and S. C. Gandevia, "Constraints for control of the human hand.," J Physiology (Lond), vol. 589, no. Pt 23, pp. 5583-5593, 2011.

[2] J. J. Belić and A. A. Faisal, "Decoding of human hand actions to handle missing limbs in neuroprosthetics," Frontiers in Computational Neuroscience, vol. 9, 2015.

[3] A. Faisal, D. Stout, J. Apel, and B. Bradley, "The manipulative complexity of lower paleolithic stone toolmaking," PLoS ONE, vol. 5, no. 11, e13718, Nov. 2010.
[4] R. W. Young, "Evolution of the human hand: the role of throwing and clubbing," J. Anatomy, vol. 202, no. 1, pp. 165-174, 2003.

[5] R. Ma and A. Dollar, "On dexterity and dexterous manipulation," vol. 15, pp. 1-7, 2011.

[6] S. K. Kim et al., "Object manipulation in 3d space by two cone-shaped finger robots based on fingerthumb opposability without object sensing," IEEE ICRA, pp. 5136-5141, 2012.

[7] C. Borst, M. Fischer, S. Haidacher, H. Liu, and G. Hirzinger, "Dlr hand ii: Experiments and experience with an anthropomorphic hand," IEEE ICRA, vol. 1, pp. 702-707, 2003.

[8] M. Chalon et al., "The thumb: Guidelines for a robotic design," IEEE Intelligent Robots and Systems (IROS), pp. 5886-5893, 2010.

[9] A. Bicchi, "Hands for dexterous manipulation and robust grasping: A difficult road toward simplicity," IEEE Robotics \& Automation (ICRA), vol. 16, no. 6, pp. 652-662, 2000.

[10] A. Dragan and S. Srinivasa, "Integrating human observer inferences into robot motion planning," $\mathrm{Au}$ tonomous Robots, vol. 37, no. 4, pp. 351-368, 2014.

[11] M. H. Schieber and M. Santello, "Neural control of movement hand function: peripheral \& central constraints ...," J Appl Phys, pp. 2293-2300, 2004.

[12] T. Feix et al., "A comprehensive grasp taxonomy," in RSS: Workshop on Understanding the Human Hand for Advancing Robotic Manipulation, 2009.

[13] C. R. Ethier and C. A. Simmons, Introductory Biomechanics. Cambridge University Press, 2007.

[14] M. V. Liarokapis et al., "Quantifying anthropomorphism of robot hands," IEEE ICRA, pp. 2041-2046, 2013.

[15] J. T. Belter et al., "Mechanical design and performance specifications of anthropomorphic prosthetic hands: a review.," J Rehabil Res Dev, vol. 50, no. 5, pp. 599-618, 2013.

[16] A. A. Thomik et al., "Towards sparse coding of natural movements for neuroprosthetics and brain-machine interfaces," in NER, 2015, IEEE, 2015, pp. 938-941.

[17] H. Yousef et al., "Tactile sensing for dexterous in-hand manipulation in robotics a review," Sensor Actuat A: Phys, vol. 167, no. 2, pp. 171-187, 2011.

[18] Z. Xu and E. Todorov, "Design of a highly biomimetic anthropomorphic robotic hand towards artificial limb regeneration," IEEE ICRA, 2016.

[19] S. J. Bensmaia and L. E. Miller, "Restoring sensorimotor function through intracortical interfaces: Progress and looming challenges," Nature Reviews Neuroscience, vol. 15, no. 5, pp. 313-325, 2014.

[20] C. Konnaris et al., "Sparse eigenmotions derived from daily life kinematics implemented on a dextrous robotic hand," in IEEE Biorob, IEEE, vol. 6, 2016. 